\title{
Energy loss spectroscopic profiling across linear interfaces: the example of amorphous carbon superlattices
}

V. Stolojan ${ }^{1, *}$, P. Moreau ${ }^{2}$, S. J. Henley ${ }^{1}$, M. J. Goringe ${ }^{3}$, S.R.P. Silva ${ }^{1}$

${ }^{1}$ Advanced Technology Institute, University of Surrey, Guildford, GU2 7XH, UK.

${ }^{2}$ Institut des Matériaux Jean Rouxel, Université de Nantes-CNRS UMR6502, 44322 Nantes, France

${ }^{3}$ School of Engineering, University of Surrey, Guildford, GU2 7XH, UK.

\section{ABSTRACT}

Energy loss spectroscopic profiling is a way to acquire, in parallel, spectroscopic information across a linear feature of interest, using a Gatan Imaging Filter (GIF) fitted to a Transmission Electron Microscope (TEM). This technique is capable of translating the high spatial resolution of a bright field image into a sampling of the spectral information with similar resolution. Here we evaluate the contributions of chromatic aberration and the various acquisition parameters to the spatial sampling resolution of the spectral information and show that this can reach $0.5 \mathrm{~nm}$, in a system not ordinarily capable of forming electron probes smaller than $2 \mathrm{~nm}$. We use this high spatial sampling resolution to study the plasmon energy variation across amorphous carbon superlattices, in order to extract information about their structure and electronic properties. By modelling the interaction of the relativistic incident electrons with a dielectric layer sandwiched between outer layers, we show that, due to the screening of the interfaces and at increased collection angles, the plasmon energy in the sandwiched layer can still be identified for layer thicknesses down to $5 \AA$. This allows us to measure the change in the well bandgap as a function of well width and to interpret it in terms of the changes in the $\mathrm{sp}^{2}$-fractions due to the deposition method, as measured from the carbon K-edges, and in terms of quantum confinement of the well wavefunction by the adjacent barriers. 


\section{Introduction}

As the components of electronic devices reduce in size, there is a need for the quantitative analysis of the properties of their constituents on the sub-nanometre scale in order to understand, model and engineer future generations of devices. Electron spectroscopy in an electron microscope is in the unique position of providing this information with sub-nanometre spatial resolution, beyond the surface of a material [1]. It has already been used successfully to answer essential questions, such as what minimum thickness is required for the $\mathrm{SiO}_{2}$ insulator in gated transistors so that it still remains insulating [2].

In most cases, the electronic structure is sampled on the subnanometre scale by forming a small-sized probe and serially acquiring spectra across features of interest. The recent advances in the correction of the spherical aberration of electron microscopes have opened the way for the sampling of the electronic structure on the sub-angstrom scale [3-5].

When the formation and scanning of a subnanometre-sized probe are not available, spatially resolved electron energy loss spectral information can still be acquired, for linear features of interest, using a Gatan Imaging Filter in a Transmission Electron Microscope. This method, denoted Energy Loss Spectroscopic Profiling (ELSP), is capable of translating the subnanometre spatial resolution achieved in a high-resolution electron microscope image into high spatial sampling resolution (in the dimension normal to the linear interface) of the spectral information [6-11]. The parallel acquisition of the energy and spatial dimensions in ELSP offers the advantage of an independent definition of the origin and scale of the spatial dimension, which is not directly available in a serially acquired spectral set. This is because, when acquiring a spectrum with a small probe, the position at which the spectrum is acquired on the sample may drift. This may be particularly important when a series of spectra is acquired in spot mode across a feature and the position of each spectrum across the spatial dimension is inferred linearly from taking images at the beginning and at the end of the series. This is not necessary for ELSP as the entire spatial dimension is collected for each image, and the position of each line spectrum is thus known independently of the whole series of ELSP images. Common to both methods, the spatial dimension is calibrated using a feature of known dimensions, present in normal images as well as in the ELSP images.

To explore the spatial sampling resolution limits of ELSP, an investigation of the effects of chromatic aberration and data processing technique is needed.

As the limits of stability and resolution are being pushed constantly, there has been a recent renewed interest in using the most readily available and intense energy loss signal, the 
plasmon excitation, to characterise the electronic structure of samples [12-14]. The crucial question regarding this excitation is what its spatial extent is. By its nature, being a collective mode of excitation, the bulk plasmon is delocalised to a few nanometers. However, there is a constant stream of articles over the years that have reported information obtained from plasmons with high spatial resolution [15-22]. In most cases, the demonstration of this high spatial resolution involved the presence of a thin layer and/or the use of particular experimental conditions (namely a collector aperture displaced to large scattering angles) when studying an interface. Therefore, investigating the limits to which sub-nanometre spatial resolution with plasmons is definable only in the case of interfaces, and particularly very thin 'sandwiched' layers, is required.

Superlattices are technologically very important structures that utilise quantum confinement and tunnelling to increase the speeds of operation of devices [23]. The bandgap of amorphous carbon can vary between $\sim 1$ to $\sim 4 \mathrm{eV}$ [24] depending on the deposition conditions, such as the bias voltage in chemical vapour deposition or the laser power in pulsed laser deposition. By varying such a parameter cyclically, superlattices can be deposited cheaply, over large areas and on temperature-sensitive substrates, consisting of what is essentially the same material [25]. Even though the layers are the same material with different densities, they can still be distinguished in bright field images even when there is as little as a $10 \%$ change in the $\mathrm{sp}^{2}$ fraction (albeit at large defoci) [26]. Complicated optical measurements, which rely on the accurate simultaneous knowledge of several parameters, were used to measure the bandgap confinement and the tunnelling effective mass. As the plasmon energy depends on the bandgap, ELSP is in the position to deliver similar information on the nanoscale. However, as the bandgaps in amorphous carbon materials are directly related to the $\mathrm{sp}^{2}$-fractions, changes in bangap energies due to quantum confinement can only be measured in cases where there are no changes in the bonding, or they are accounted for. Again, ELSP can offer that information with relatively high spatial resolution, depending on the spatial drift of the sample.

In Section 2, we first review the ELSP method and the way the spatially resolved information is produced and recorded. Section 3 studies the spatial resolution of the low-loss ELSP images by first recognising two components: the spatial sampling resolution and the plasmon delocalisation. Section 3.1 evaluates the spatial sampling resolution in terms of the instrument stability, the recording and averaging methods (for good statistics) and the effect of chromatic aberration. In Section 3.2 relativistic simulations are used to model the response of a sandwiched layer as a function of its width and to study the conditions over which subnanometre spatial localisation can be defined. Section 4 summarises the experimental and data 
analysis procedures whilst Section 5 demonstrates the capability of ELSP on amorphous carbon superlattices, and then we conclude with a discussion of the main results in Section 6.

\section{Energy Loss Spectroscopic Profiling (ELSP)}

ELSP refers to a mode of acquisition of spatially resolved spectra across linear interfaces, using a Gatan Imaging Filter (GIF) [11]. One way to describe the data provided at the spectrometer's slit is through a three-dimensional set, where two dimensions are the spatial dimensions of the projected image of the sample, and the third dimension is the energy lost through inelastic interactions by the incident electron beam to the sample. The various modes of acquisition (energy loss spectra, energy filtered images) then are just ways to sample and project the 3D data set onto the $2 \mathrm{D}$ detector which is the charge-coupled-device (CCD). To obtain an energy filtered image, the mechanical slit is employed to select a section of the 3D data set through an energy interval, and then project it along the energy axis onto the CCD. An energy loss spectrum is the 3D data set integrated in the two spatial directions. The way this is performed in the CCD is first along one direction using the quadrupoles. The $2 \mathrm{D}$ result, with one remaining spatial dimension and the energy loss dimension is then recorded at the CCD and then the spatial dimension is integrated by binning on the CCD. However, if this last step is by-passed, the image recorded at the CCD still retains one of the spatial dimensions of the projected image of the sample. In the particular case of linear interfaces, if these are oriented along the axis integrated by the quadrupoles (Fig. 1a), it follows that the remaining spatial dimension recorded at the CCD represents linear positions across this interface (Fig. 1b, 1c).

(Insert Figures 1a 1b 1c about here)

In other words, it is similar to forming a line probe parallel to the linear feature of interest (as opposed to a circular spot) in a STEM and then scanning across the interface. The difference comes from the circular spectrometer entrance aperture, which results in the "STEM line probe" 's length variation as limited by the aperture. However, the parallel nature of the acquisition of the spatial dimension ensures one has an independent measure of the spatial dimension, concomitant with the acquisition of the spectral information. A line profile at constant zero loss energy in Fig. 1c is identical, to within a scaling factor, to the line profile normal to the interfaces in Fig. 1a, integrated within an area defined by the size of the spectrometer's collector aperture. Compared to a line scan in STEM mode, the spatial dimension is thus sampled for each data set collected, as opposed to just at the beginning and the end of the data series. However, the lower equivalent current density contained in the "linear probe" in ELSP means that its effectiveness is 
restricted to low-energy loss spectra and to relatively lower energy core edges (carbon, nitrogen, oxygen). At the higher energy-loss edges, the effectiveness of ELSP is limited by the stability of the microscope-spectrometer system (sample drift, high-tension stability, etc.). In the case of field-emission microscopes however the increased current density allows for shorter exposure times; coupled with generally better stabilities, the analysis of higher edges (eg. Fe and Ni Ledges) is easily achieved $[10,11]$.

Nevertheless, this technique can offer sub-nanometre-resolved spectral sampling across linear interfaces, in microscopes (such as the one used here) not capable of forming probes theoretically smaller than $2 \mathrm{~nm}$, and even then without reasonable current density for good signalto-noise ratios in spectral information.

\section{Spatial resolution of ELSP}

We can divide the discussion on spatial resolution into two distinct issues: the first relates to the technique's ability to sample the spatial dimension with sub-nanometre resolution, and the second issue relates to the spatial (de)localisation of the inelastic event studied. The spatial sampling resolution is defined by mechanical and drift stability during one exposure, the accurate alignment between several exposures and chromatic aberration and spatial resolution (spherical aberration) of the microscope. The (de)localisation of inelastic events is a hotly debated subject [27, 28], but generally one can say that core-losses are localised to angstroms, whilst valence losses, such as bulk plasmons, have extinction distances of the order of nanometres.

\subsection{Spatial sampling resolution}

The spatial sampling is defined by the microscope's magnification and the strength of quadrupole 5 (Q5) of the GIF [7]. The first selects the length of the spatial dimension sampled, and the second selects the number of CCD pixels over which this dimension is mapped. In our particular set-up, the $\sim 1024$ pixels of the projected image (Fig.1a) are mapped to $\sim 550$ pixels of the ELSP image (Fig. 1b, 1c), allowing for a spatial sampling of $\sim 0.7 \AA /$ pixel.

When several ELSP images are averaged for good signal-to-noise statistics, the spatial sampling resolution can be degraded by the sample drift/instrument stability within one exposure time interval, and further worsened by the ability to align several images to within a pixel. The alignment is more easily done for low energy loss spectra, where the profile across the zero-loss energy can be used effectively. Figure 2 traces the drift of the high voltage and the sample drift 
as a function of time, as measured from a set of low loss ELSP images collected with exposure times of $0.5 \mathrm{~s}$, with $4.5 \mathrm{~s}$ between exposures (needed for the transfer of data from the CCD to the computer's hard disk). The drift values here are representative of the microscope used (Philips CM200 Supertwin TEM, 200kV accelerating voltage, $\mathrm{LaB}_{6}$ electron source) and partly of the spatial and energy dispersions used $(\sim 0.7 \AA /$ pixel, $\sim 0.1 \mathrm{eV} /$ pixel respectively).

(Insert Figure 2 about here).

For the experiments described here, the average spatial drift is $\sim 0.06 \mathrm{~nm} / \mathrm{s}$. This means that within the time of a low loss ELSP acquisition the sample drift was, on average, equivalent to one pixel and unlikely to affect the ultimate spatial resolution. The situation changes for highloss ELSP images, where exposure times of the order of 3-5s are needed (for C K edges, for example). For this particular dispersion, the biggest contributor to the degradation of the spatial sampling resolution compared to that of the normal TEM image is our ability to align images with respect to each other (detailed in Section 4). For the low loss ELSP, this is to within $\sim 2$ pixels, which translates to an average position uncertainty of 0.707 x 4 pixels x $0.7 \AA /$ pixel $\approx 2 \AA$. The effect of chromatic aberration is expressed as:

$$
\Delta \mathrm{f}=\mathrm{C}_{\mathrm{C}} \frac{\Delta \mathrm{E}}{\mathrm{E}_{0}}
$$

where $\Delta \mathrm{f}$ is the amount of defocus associated with the energy lost $\Delta \mathrm{E}$, given the incident energy $\mathrm{E}_{0}$ and chromatic aberration coefficient $\mathrm{C}_{\mathrm{C}}$. This is seen in Fig. 3, which is a cut-out section of Fig. $1 c$ at the superlattice-mounting glue interface.

\section{(Insert Figure 3 about here)}

It can be seen that, at this interface, there is a contrast change along the energy axis (Fig. 3), which is caused by the defocus due to chromatic aberration of the microscope-spectrometer system. Following the contrast change along the axis, we can approximately identify gaussian focus in the region of the amorphous carbon plasmon energy (indicated by the arrow in Fig. 3); this position changes when varying the defocus, according to equation 1. Practically, this means that one must always take into account the defocus due to chromatic aberration when collecting and interpreting the spatial distribution of energy loss signals. For our experimental set-up, if the drift tube is used to acquire the carbon K-edge a large $(\sim 2 \mu \mathrm{m})$ overfocus is needed to bring the structure back into focus (and also verifying the value of the experimental $C_{C}$ calculated).

Therefore, the way to change the portion of the spectrum recorded without the need for applying 
this large defocus is to change the accelerating voltage, as is done when performing energyfiltered TEM [11]. To some extent this could affect all microscope-spectrometer systems, when changing the drift tube voltage to access various energy intervals of the energy loss scale. To continue this argument, we present at this stage the plasmon energy profiles across an amorphous carbon superlattice measured at different defoci (Figure 4), deferring discussion of the data processing and analysis until later.

(Insert Figure 4a,b about here)

The inset in Figure 4a shows that the measured plasmon energies at the Si substrate (on the left-hand-side of the inset) and at the mounting glue (on the right-hand-side of the inset) do not change significantly with the change in focus; therefore we conclude that the changes observed across the superlattice are not caused by the amount of defocus applied but possibly by some interference of the inelastic electrons.

We can see that, with overfocus, the plasmon traces across the superlattice lose their definition and there is a significant reduction in the plasmon energy difference between barrier and wells, down to its disappearance for $+1040 \mathrm{~nm}$ overfocus. In the case of underfocus, the effects are more attenuated, where there is a gradual decrease in the energy difference between barrier and wells in the plasmon energy profiles together with a lowering of the average plasmon energy. This asymmetry in the plasmon energy difference is similar to observations using Fresnel fringe analysis when the inelastic contributions have not been filtered out (also known as in-line holography)[29,30]. Figure $4 \mathrm{~b}$ summarises the changes in contrast, measured as the difference between barrier and well intensity, normalised to their sum, as a function of defocus. It shows that, up to approximately 500nm of defocus, there is little effect on the plasmon energy difference or spatial sampling resolution of the measured plasmon energy trace across the superlattice, which translates to an energy range of (eq. 1) 70eV. For energy losses beyond this range, the effect of chromatic aberration (translating into an increasing amount of defocus with increasing energy loss) can be easily minimised or cancelled either by a small amount of overfocus or by changing the high-tension voltage (and not the drift tube voltage) when accessing higher energy intervals of the spectra.

(Insert figure 5 about here)

In figure 5, we interpret the change in the plasmon energy difference as a loss of spatial sampling resolution due to defocus caused by the chromatic aberration. Therefore, we are comparing the experimental profiles for a range of defoci with the experimental profile at $0 \mathrm{~nm}$ 
defocus convoluted with a Gaussian of full-width-at-half-maximum (FWHM) varying from $0.5 \mathrm{~nm}$ to $3 \mathrm{~nm}$, representing the worsening of the spatial sampling resolution. We note very little difference betweem the $0 \mathrm{~nm}$ profile convoluted with a $0.5 \mathrm{~nm}$ FWHM Gaussian and the plasmon energy profile at $-347 \mathrm{~nm}$. This means that, when the structure is in focus at $0 \mathrm{eV}$ loss, the effective spatial sampling resolution due to chromatic aberration defocus for the plasmon energy trace is no worse than $5 \AA$. To remember the context, at the same spot size used when acquiring ELSP images, the smallest probe diameter is $\sim 50 \mathrm{~nm}$ (compared to the $0.5 \mathrm{~nm}$ resolution in ELSP images). Even in nanoprobe mode, the best probe diameter obtainable is $2 \mathrm{~nm}$, with a $20 \mu \mathrm{m}$ diameter condenser aperture (as opposed to $100 \mu \mathrm{m}$ for ELSP). This will constrain the stability conditions imposed on the microscope due to acquisition times increasing by an order of magnitude.

By comparison, the plasmon energy profiles at $116 \mathrm{~nm}$ overfocus and $-810 \mathrm{~nm}$ defocus have a spatial sampling resolution degraded to $1 \mathrm{~nm}$, whilst at $347 \mathrm{~nm}$ overfocus it is $2 \mathrm{~nm}$ and $3 \mathrm{~nm}$ at $578 \mathrm{~nm}$ overfocus.

We believe that, although the effects are relatively small over large energy ranges, when comparing spatially-resolved chemically-selected profiles across various structures, changing the drift tube voltage should be avoided, or the chromatic aberration should be corrected for.

In conclusion, the spatial sampling resolution limit in ELSP is dictated primarily by the spatial stability of the sample-microscope-spectrometer system, and by the spatial dispersion used. With care, this spatial sampling resolution limit approaches $\sim 0.5 \mathrm{~nm}$ in our system, and can probably be pushed lower in more stable environments, up to the resolution attained in a TEM image. Chromatic aberration does play a significant role when large energy intervals are involved, and/or the drift tube is used to access various energy ranges of the spectra.

\subsection{Plasmon localisation in 'sandwich' structures}

As we have established that the recording system (microscope-spectrometer) is capable of sampling the spatial dimension with sufficient resolution, we now turn our attention to the physical extent of the event studied. In the case of core-loss edges, such as carbon K-edges, it is generally accepted that the event is strongly localised to the dimensions of the 1s Bloch state (for the $\mathrm{C}$ K-edge), convoluted by the probe and detector functions [27, 28]. In most cases, the probe

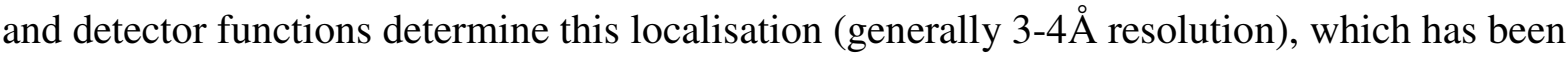
extensively studied and discussed $[31,32]$. Due to our instrument's characteristics, we shall concentrate more on the low energy-loss portion of the spectra and particularly on the plasmon energy. Being the inelastic event with the highest scattering cross-section, it reduces drastically 
the mechanical and electric stability constraints imposed on the system. Besides, this is the signal recorded closest to the conditions and exposure times at which the energy calibration and the measurement of the energy resolution are performed. The experimental question we are asking is, if we are to study the changes in plasmon energy when decreasing the filler (either a barrier or well) width as due to a change in the bandgap energy caused by quantum confinement, what is the influence of the interfaces and of the outer layers on the plasmon energy measured in the middle of the filler layer?

A bulk plasmon itself is delocalised to several nanometres [20, 27, 33], but this is generally shown to be limited to values smaller than the adiabatic cut-off criterion and even subnanometre dimensions near interfaces. In general, there are two situations (which can also be concurrent) which help with the sub-nanometre localisation of plasmons: one is the screening effect of the interface plasmons [20 and references therein] and the other is the localisation of the signal at large collection angles [27, 34].

The screening effect of the interface plasmons can be viewed as a consequence of the 'begrenzung' effect [20]. It happens at an interface between two media of dielectric functions $\varepsilon_{\mathrm{A}}$ and $\varepsilon_{\mathrm{B}}$ and refers to the replacement (as opposed to the superposition) of the contribution from dielectric medium A to the scattering cross section, when nearing the interface, by the interface plasmon [19]. This effectively leads to a screening of the contributions of the adjacent dielectric media from each other by the interface plasmon.

Using relativistic dielectric theory for interfaces [35], we model two simple structures based on the experimental superlattice measured in Fig.4; one is a barrier-well-barrier and the other is a well-barrier-well sandwich. Experimental low-loss spectra extracted from a barrier and a well were used to obtain the barrier and well dielectric functions respectively. A KramersKronig-Analysis (KKA) was used taking the value of 2.4 for the refractive index in the normalisation procedure $[1,36]$. KKA is usually very dependent on the extraction method (removal of the zero loss, normalisation, etc.). In the case of the plasmon peak around $29 \mathrm{eV}$ and in the context of a comparison of different sandwiches, the calculation results were in fact almost insensitive to the extraction parameters. These dielectric functions are inputs to a computer programme with the sandwich thickness, the probe position and the collection angles as the main parameters [37]. It should be noted that in the formulae used here, the transferred momentum is only limited in the direction parallel to the interfaces. However, the general trends given on the spectrum dependency on momentum transfer values are still valid.

Figure 6 summarises the changes to the plasmon energy in the middle of the filler layer due to the interfaces with the outer layers. As the filler's width decreases, the plasmon energy (filled squares) begins to shift towards the plasmon energy of the outer layers. By the time the 
filler has reached $1 \mathrm{~nm}$ width, the interfaces contribute significantly to the value of the plasmon energy. Even if the filler layer is still 'visible' in the plasmon energy trace down to $\sim 0.5 \mathrm{~nm}$, it may be difficult to separate between the energy contribution of the interface plasmons and the energy change due to quantum confinement. However, if the collector angle is increased (in ELSP, the largest available collector aperture is used), the plasmon energy gets closer to the filler's value, hence diminishing the influence of the outer layer interfaces. From the formulae in Appendix B of Moreau et al [19], it is easily shown that the limit for large transferred momenta is the very local spectrum and, consequently, an increased aperture size (or a displaced aperture) favours the localization of the signal (filled circles - Fig. 6)

As a result of this and of the 'begrenzung' effect, we are able to measure plasmons and interpret their energies on angstrom scales. It must be noted that here there is a physical limit on the thickness of the filler layer so that one can dielectrically define it as a layer with two surfaces, as opposed to a 'thick' interface between two media [38].

\section{Experimental Procedure}

The amorphous carbon superlattice samples were deposited by pulsed laser ablation of a 99.999 \% pure pyrolytic graphite target (Kurt J. Lesker) using a Lambda-Physik LPX 210i excimer laser producing pulses of $25 \mathrm{~ns}$ duration, at $248 \mathrm{~nm}$. The growth chamber was evacuated using a turbo-molecular pump to $9 \times 10^{-8}$ Torr. The laser pulses were focused onto the rotating target, producing a fluence of $\sim 4 \mathrm{~J} / \mathrm{cm}^{2}$ and $\sim 19 \mathrm{~J} / \mathrm{cm}^{2}$ for the well and barrier layers, respectivley. The fluence used could be varied rapidly by deploying a series of pneumatically controlled attenuators, which assisted in the production of sharp interfaces. The ablated material was deposited onto Si (100) substrates located $6 \mathrm{~cm}$ from the target. The precise growth rate at each fluence was determined by measuring the thickness, by ellipsometry and profilometry, of a film deposited with 3000 laser shots. Thinner layers could then be deposited by varying the number of laser shots accordingly.

Three samples were prepared: 333, consisting of five barriers and four wells nominally $3 \mathrm{~nm}$ wide; 444 , consisting of four barriers and three wells nominally $4 \mathrm{~nm}$ wide and 888 , consisting of two barriers and one well nominally 8nm wide. Cross-sectional TEM samples were prepared by mechanical grinding and polishing, followed by precision ion beam polishing (Gatan PIPS $^{\text {TM }}$ operated at $4.5 \mathrm{keV}, 4.5^{\circ}$ thinning angle, 20 minutes thinning). The ion beam polishing is not generally suited for samples that have not been previously annealed as it may change the $\mathrm{sp}^{2} / \mathrm{sp}^{3}$-type bonding of the barriers and the layer. The ion beam is expected to affect mostly the high-sp ${ }^{3}$-bonded barrier layers and lead to their amorphisation. However, our results do not show 
a statistically significant change in the value of the measured $\mathrm{sp}^{2} / \mathrm{sp}^{3}$ ratio for all the samples studied (see section 5), therefore we conclude that, if modified by the ion beam, all samples are affected in a very similar way, so that comparisons of valence and $\mathrm{sp}^{2} / \mathrm{sp}^{3}$ compositions are still valid.

For each sample, 40 low loss ELSP images were collected, with $0.5 \mathrm{~s}$ exposure times. The carbon K-edge ELSP images required longer exposure times (2s) and a more intense beam for good statistics, hence only $\sim 5$ spectra were collected from one site. Longer exposures under an intense electron beam resulted in the amorphisation of the barrier layers, as seen by a loss of contrast in the bright field image of the superlattice's layers. We also collected 40 ELSP ( 0.5 s exposure) images with the beam unscattered by the sample (passing through a hole) for a measure of the point-spread-function and the origin of the energy scale for each of the line spectra within an ELSP image. A further 20 energy calibration spectra ( $0.5 \mathrm{~s}$ exposure) were collected with a $40 \mathrm{~V}$ oscillation applied to the drift tube, necessary for the individual calibration of the energy scale for each of the line spectra within one ELSP image. To minimise the need for the removal of multiple scattering contributions to the low and high loss ELSP images, we restricted the measurements to regions with thicknesses below 0.5 (normalised to the mean-free-path for inelastic scattering).

Before analysis, all ELSP sets had to be aligned in energy as well as in spatial position (for the low and high loss ELSPs). The energy alignment is not difficult for the low and zero loss as the signal-to-noise ratio is very good. Therefore, either the drift measurement routine provided by Digital Micrograph ${ }^{\mathrm{TM}}$ or a comparison of the energy profiles obtained by integrating an ELSP image in the spatial direction worked well, with 1 pixel accuracies. The alignment of the spatial dimension was more complicated when using the drift measurement routine because of the relatively small dimensions of the features used for alignment and the comparatively high number of counts in the zero loss peak. Usually, the Si-superlattice interface was used for spatial drift alignment; when using the interface's fingerprint within the zero-loss peak a relatively small image area needed to be used so that the autocorrelation within the drift correlation routine would produce distinguishable results for the spatial dimension. In order to increase the precision, we selected larger areas of the ELSP images at higher energies (in the region of the $\mathrm{Si}$ and carbon plasmons) but this was done at the (smaller) expense of increasing the width of the crispening filter. Nevertheless, the larger areas used meant that we could still position ELSP images with respect to each other to within one pixel, at the most 2 pixels.

Once the data sets were aligned and averaged, each of the $\sim 550$ line spectra was analysed. In the case of the high-loss profiles, the $\mathrm{sp}^{2}$ fraction was calculated from each $\mathrm{C}$ K-edge using the 2Gaussian method, after fitting and subtraction of the power-law pre-edge backgrounds. Thus, a 
measure of the area of the $\pi^{*}$ peak is obtained which, when normalised to a $100 \% \mathrm{sp}^{2}$-bonded standard, represents the $\mathrm{sp}^{2}$-bonded fraction of carbon in the sample. Similarly, the plasmon energies were measured from each of the line spectra of the low-loss ELSP images using a modified Lorentzian curve. Note that we measured the plasmon energy as defined using the Drude-Lorentz model [1], and not the peak maxima in the spectra. The use of several tens of pixels for the fitting scales down the errors in the measurement of the plasmon energies by $\sim 1 / \sqrt{ } \mathrm{N}$, where $\mathrm{N}$ is the number of data points used. The origins of the energy scales were determined from the zero loss peaks in each of the low-loss ELSP images, as fitted using Lorentzian curves. Thus, the energy origins could be determined to within $\sim 20 \mathrm{meV}$, whilst the plasmon energies were determined to within $\sim 10 \mathrm{meV}$. Furthermore, the plasmon energies across the superlattices were corrected for the individual energy calibrations.

As a final test, Figure 7 shows a comparison of the measured plasmon energy across a test superlattice (10 wells and 11 barriers, 6 and $8 \mathrm{~nm}$ wide respectively) with the thickness across the sample, as determined from the low loss spectra [1] (normalised to the mean-free-path for inelastic scattering). The thickness gradient is $\sim 4^{\circ}$ degrees, similar to the ion beam-milling angle. However, the plasmon energy trace shows little dependence on the either the gradient or the preferential etching visible at the higher thicknesses. The plasmon energy difference between barriers and wells remains largely constant and is thus a measure of the differences in the electronic structures of the barriers and wells.

(Insert Figure 7 about here)

\section{Morphologies and Electronic Structures of Amorphous Carbon Superlattices}

A defocused energy-filtered image of Sample 888 (Fig. 9a) showed that, whilst the well was $\sim 8 \mathrm{~nm}$, the barriers were $\sim 10 \mathrm{~nm}$. Figure $9 \mathrm{~b}$ shows the measured plasmon energy and $\mathrm{sp}^{2}$ fraction profiles, estimating the barriers at $\sim 20 \% \mathrm{sp}^{2}$ content and the wells at $\sim 55 \% \mathrm{sp}^{2}$ content.

(insert fig 8a, 8b, 9a, 9b, 10a, 10b about here)

The defocused bright field images of superlattices 444 and 333 reveal that the barrier and well dimensions are much closer to the nominal widths ( 4 and $3 \mathrm{~nm}$ respectively), but there is a systematic shrinking of the well layers in comparison with the barrier layer. This could be because of the higher stresses associated with the more diamond-like barrier layers, which induces their slight expansion at the expense of the wells. The $\mathrm{sp}^{2}$ fraction traces though reveal a possible limitation of this growth technique, where the wells now are no longer as graphitised as 
the well in Sample 888. The $\mathrm{sp}^{2}$ fractions of the wells have now reduced to $40 \%$, whilst the $\mathrm{sp}^{2}$ fraction of the mounting glue and the barriers are relatively unchanged. This shows that the change in the $\mathrm{sp}^{2}$ fraction of the wells is not due to the ion beam polishing and/or a low spatial sampling resolution (because the barrier plasmon energies and $\mathrm{sp}^{2}$ fractions are unchanged). One explanation is that, as we increase the laser power to deposit a barrier layer on top of a well layer, the well layer is more stressed so that its width is reduced and it becomes more diamondlike.

A closer comparison of the plasmon energy traces for superlattices 444 and 333 (figure 11) shows an increase in the well plasmon energy with decreasing well width.

(Insert Figure 11 about here)

For a material with a bandgap $E_{g}$ we can write the plasmon energy $E_{P}$ as [1]:

$$
\mathrm{E}_{\mathrm{P}}^{2}=\hbar^{2} \frac{\rho_{\mathrm{el}} \mathrm{e}^{2}}{\varepsilon_{0} \mathrm{~m}_{\mathrm{e}}^{*}}+\mathrm{E}_{\mathrm{g}}^{2}
$$

representing a bound oscillator, with the 'free-electron gas' plasmon energy given by the first term on the right-hand-side of equation $2\left(\hbar=\right.$ reduced Planck's constant, $\mathrm{e}=$ electron charge, $\varepsilon_{0}$ $=$ permittivity of vacuum, $\rho_{\mathrm{el}}=$ density of valence electrons and $\mathrm{m}_{\mathrm{e}}{ }^{*}$ the effective mass of the electrons taken at dipole transitions). The bandgap $\mathrm{E}_{\mathrm{g}}$ is not the optical bandgap but rather the Penn gap, a parameter introduced to adjust the free electron model at small values of the wave vector $\mathrm{k}$, for Bragg reflections and Umklapp processes (non-dipole transitions) [39]. The Penn gap $\mathrm{E}_{\mathrm{g}}$ is defined through the relationship:

$$
\mathrm{n}^{2}=1+\left(\frac{\mathrm{E}_{\mathrm{p}}}{\mathrm{E}_{\mathrm{g}}}\right)^{2}
$$

where $\mathrm{n}$ is the optical refractive index. Another view of the Penn gap in carbon is as an average separation of the bonding and anti-bonding energy levels, as opposed to the difference between the highest-occupied bonding level and the lowest unoccupied anti-bonding level. Assuming the free electron value in equation 2 does not change between the 444 and 333 superlattices, and using n=2.2 for the well in superlattice 444, we find the Penn gap (Eq. 3) $E_{g} 444$ $=14.12 \mathrm{eV}$. Using equation 2 we derive the free electron value, which we can then use to 
determine the Penn bandgap in superlattice $333 \mathrm{E}_{\mathrm{g} 333}=15.09 \mathrm{eV}$. There is a $1 \mathrm{eV}$ average Penn bandgap increase, which results from the increased quantum confinement with decreasing well width in superlattice 333 .

\section{Conclusions}

We have shown that, in the case of linear features, the ELSP technique is capable of transferring the sub-nanometre spatial resolution available in the normal imaging mode in a TEM to the sampling of the energy loss information acquired using a GIF. We have evaluated the effects of sample drift, image alignment and chromatic aberration and shown that with appropriate choices of microscope and spectrometer conditions, we can sample the spatial dimension across a linear interface with sub-nanometre resolutions, $\sim 5 \AA$ for the equipment and with the conditions used here. This is 1-2 orders of magnitude better than if the same microscope were used to form a small probe, with sufficient current density, and then scanned across the linear feature.

The parallel nature of the acquisition of the energy dimension and one spatial dimension offers the unique ability to independently measure and define the spatial position of energy loss spectra, allowing accurate correlations of spectral information with particular (linear) features across several samples (this is more difficult to do with probe scanning serial acquisition modes). In general, due to the low current density, the equivalent signal-to-noise factors are lower, which limits ELSP (with a $\mathrm{LaB}_{6}$ cathode) to low-energy losses and relatively low core-losses, such as the carbon K-edge (depending on the stability of a particular instrument).

We demonstrated the ability of ELSP to sample the energy loss information on subnanometre spatial scales using amorphous carbon superlattices. First we showed that plasmon energies are definable and measurable in thin sandwiched layers down to sub-nanometre dimensions, due to the effective screening of the interfaces and the low scattering probability of the interface plasmons at large angles. We then measured the carbon $\mathrm{sp}^{2}$ fraction and plasmon energy across a series of superlattices and showed that, with laser ablation, the layer morphology changes as the well layers get smaller, possibly due to the stresses caused by the more diamondlike barriers. We have also shown that, as the wells get even smaller, the well bandgap increases and have attributed this increase to quantum confinement effects. 
References

[1] R. F. Egerton, Electron Energy Loss Spectroscopy in the Electron Microscope. $\left(2^{\text {nd }}\right.$ ed., Plenum Press, New York \& London, 1996).

[2] D.A. Muller et al., Nature 399 (1997) 758

[3] M. Haider, H. Rose, S. Uhlemann, B. Kabius, K. Urban, J. Electron Microsc. 47 (1998) 395.

[4] O.L. Krivanek, N. Dellby, A.R. Lupini, Ultramicroscopy 78 (1999) 1.

[5] P.E. Batson, O.L. Krivanek, N. Dellby, Nature 418 (2002) 617.

[6] J. Bentley, I.M. Anderson, Proc. Microscopy and Microanalysis '96 (San Francisco Press 1996) 532.

[7] T. Walther, MSM Proc., IOP Conf. Ser. 164 (IOP Publishing Ltd 1999) 121.

[8] T. Walther, et al., Phys. Stat. Sol. (a) 180 (2000) 351.

[9] T. Walther, Physica B 308-310 (2001) 1161.

[10] G. A. Botton, et al., J. Appl. Phys. 91 (2002) 2921.

[11] T. Walther, Ultramicroscopy 96 (2003) 401.

[12] V. Keast, J. of Elec. Spectr. and Related Phen. 143 (2005) 99-106.

[13] R. Erni and N. Browning, Ultramicroscopy (2005) in press.

[14] S. Pokrant, M. Cheynet, S. Julian, R. Pantel, Ultramicroscopy (2005) in press.

[15] A. Howie, C. A. Walsh, Microsc. Microanal. Microstruct. 2 (1991) 171.

[16] D. Ugarte, C. Colliex, P. Trebbia, Phys. Rev. B45 (1992) 4332.

[17] M.A. Turowski, T.F. Kelly, P.E. Batson, J. Appl. Phys. 76 (1994) 3776.

[18] Z.L. Wang, Micron 27 (1996) 265.

[19] P. Moreau, N. Brun, C.A. Walsh, C. Colliex, A. Howie Phys. Rev. B56, 6774-6781 (1997).

[20] A. Howie, in Topics in Electron Diffraction and Microscopy of Materials (ed. P. B. Hirsch, Taylor and Francis, 1999) 79.

[21] T. Stockli et al., Phys. Rev. B61 (2000) 5751.

[22] N. Zabala, E. Ogando, A. Rivacoba, F. J. Garcia de Abajo, Phys. Rev. B64 (2001) art. no. 205410.

[23] B. Abeles, T. Tiedje, Phys. Rev. Lett. 51 (1983) 2003.

[24] S.R.P. Silva, G.A.J. Amaratunga, C.P. Constantinou, J. Appl. Phys. 72 (1992) 1149.

[25] S.R.P. Silva, G.A.J. Amaratunga, Rusli, S. Haq, E.K. Salje, Thin Solid Films 253 (1994) 20.

[26] C.A. Davis et al, Phys. Rev. Lett 75 (1995) 4258.

[27] D.A. Muller, J. Silcox, Ultramicroscopy 59 (1995) 195.

[28] A.R. Lupini, S.J. Pennycook, Ultramicroscopy 96 (2003) 313. 
[29] R E Dunin-Borkowski, C B Boothroyd, S J Lloyd, W M Stobbs, J. Microsc. 180 (1995) 263-276.

[30] R.E. Dunin-Borkowski, Ultramicroscopy 83 (2000) 193.

[31] N. K. Menon and J. Yuan, Ultramicroscopy 78 (1999) 185.

[32] M. Varela et al., Phys. Rev. Lett. 92, 095502 (2004)

[33] M. Inokuti, Rev. Mod. Phys. 43 (1971) 297.

[34] R. H. Ritchie, A. Howie, Phil. Mag. A58 (1988) 753.

[35] R. Garcia Molina, A. Gras Marti, A. Howie and R.H. Ritchie, J. Phys. C 18, 5335 (1985).

[36] P. Fallon, DK programme, Deconvolution \& Kramers-Kroning Analysis of EELS spectra, (Cavendish Laboratory, Cambridge, 1991)

[37] C.A. Walsh, Computer Programmes for the Calculation of Electron Energy-Loss Spectra from Interfaces Between Dielectric Media. (Cavendish Laboratory, Cambridge, 1992).

[38] V. Stolojan et al., Appl. Phys. Lett. 86 (2005) art. no. 063112.

[39] D.R. Penn, Phys. Rev. 128 (1962) 2093. 
Figures $1 \mathrm{a}, \mathrm{b}, \mathrm{c}$

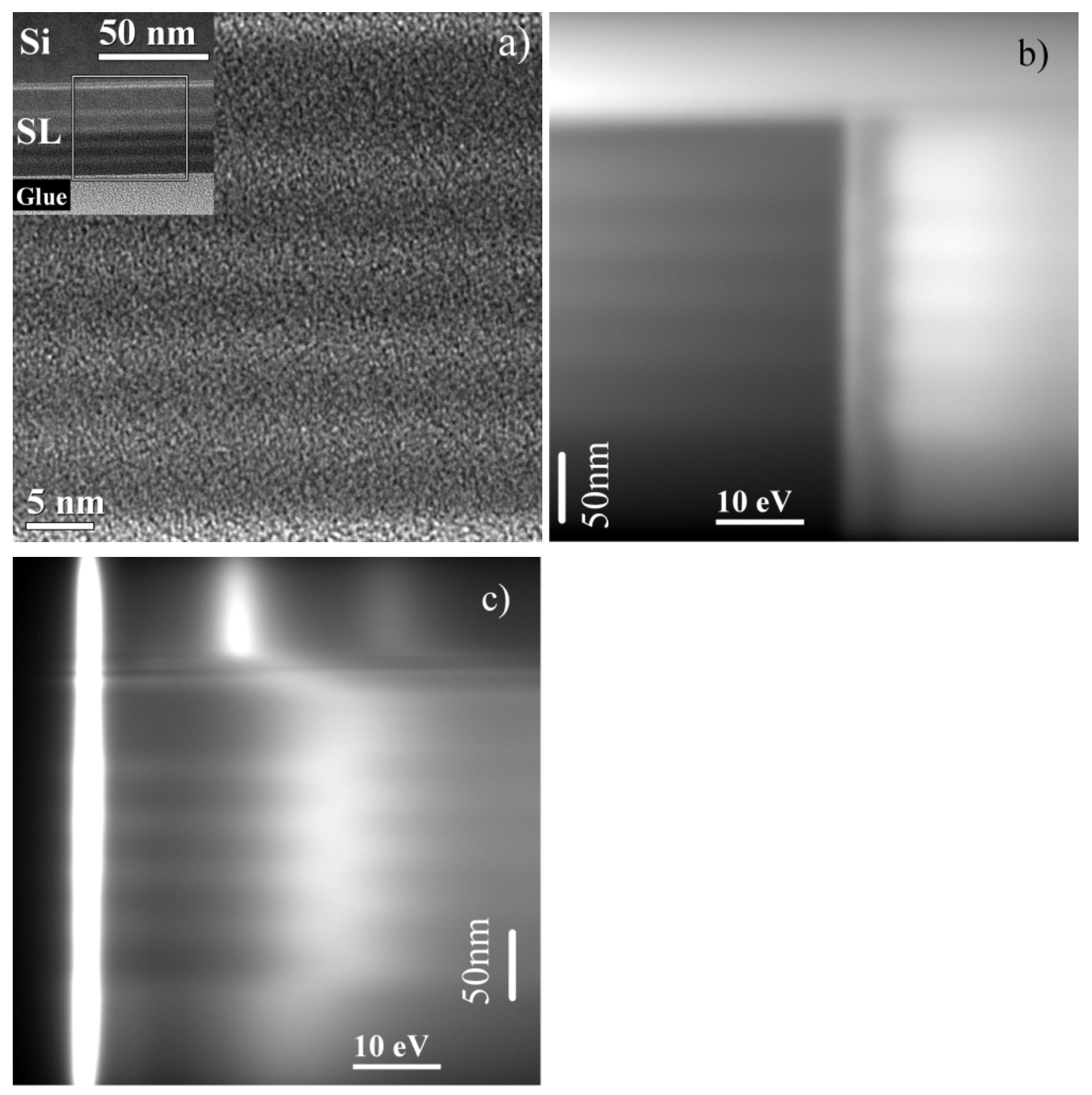


Figure 2

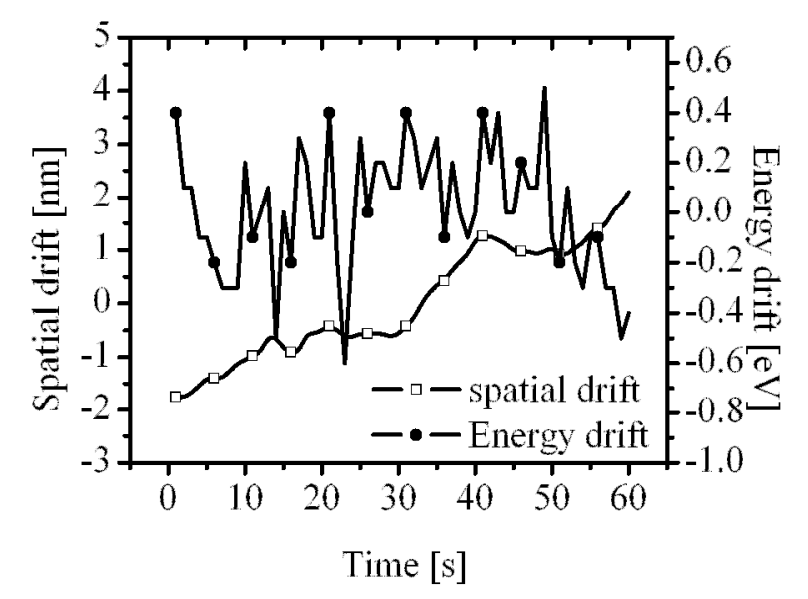

Figure 3

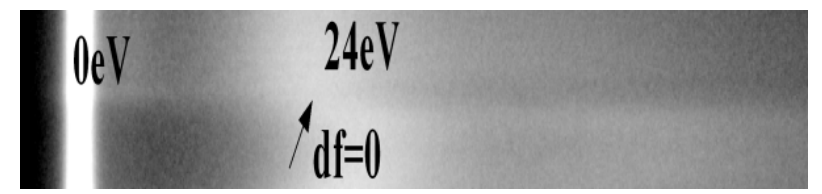


Figure 4 a,b
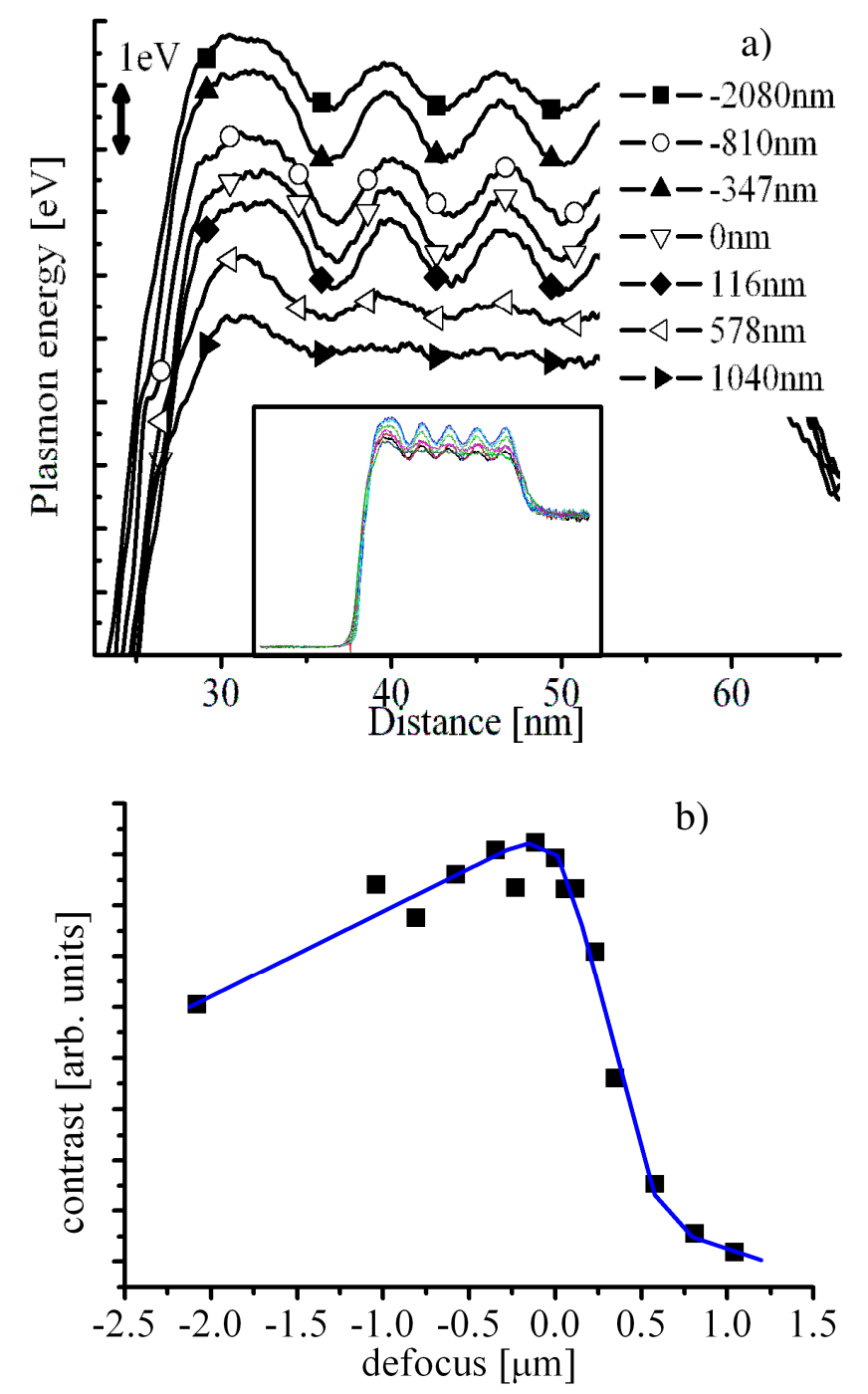
Figure 5

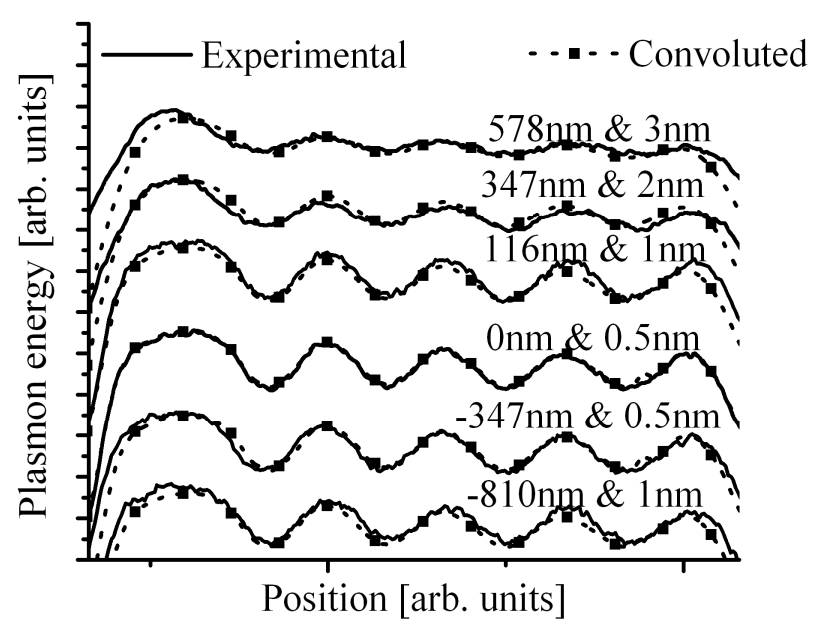

Figure 6

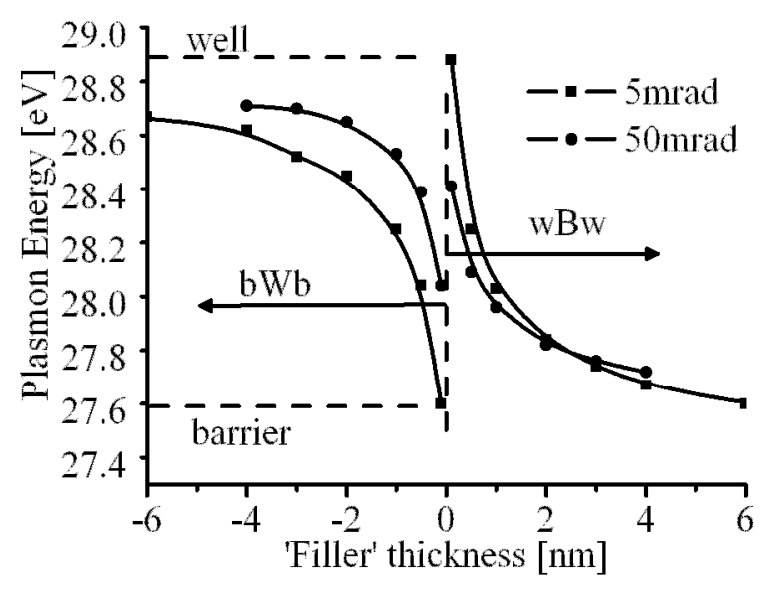


Figure 7

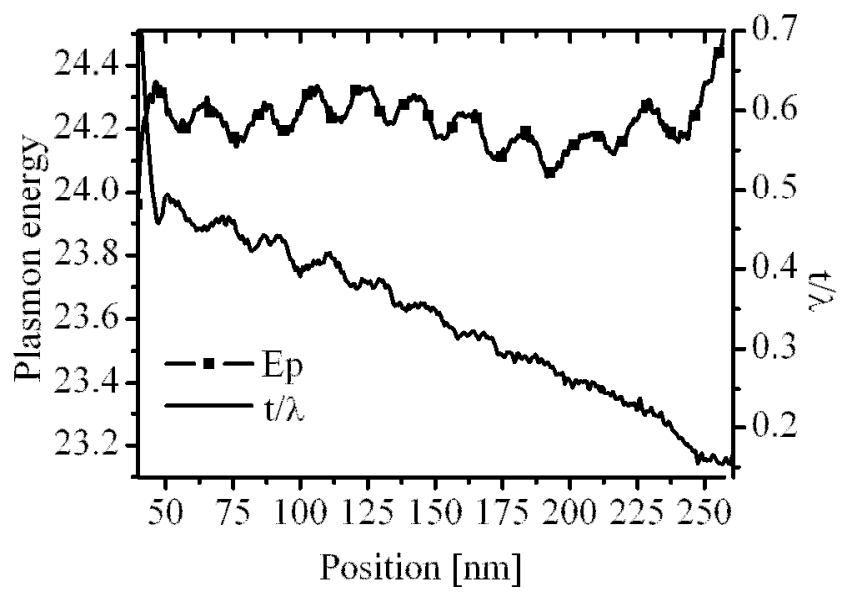


Figure 8a,b
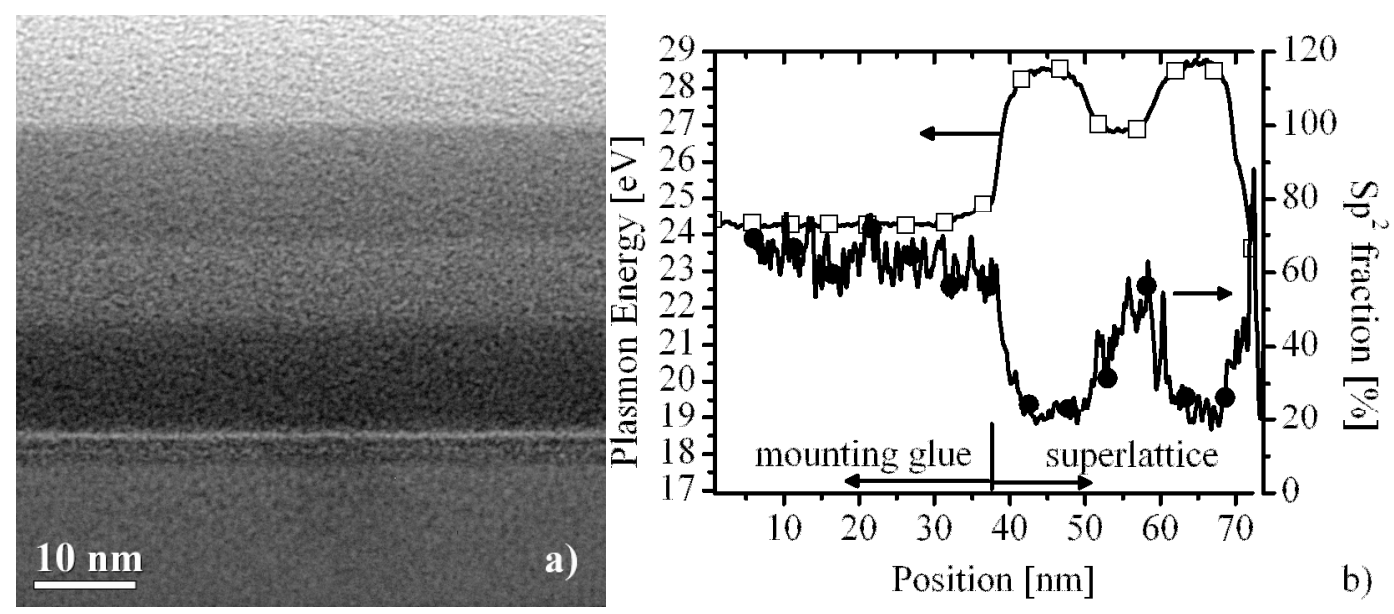

Figure 9a,b
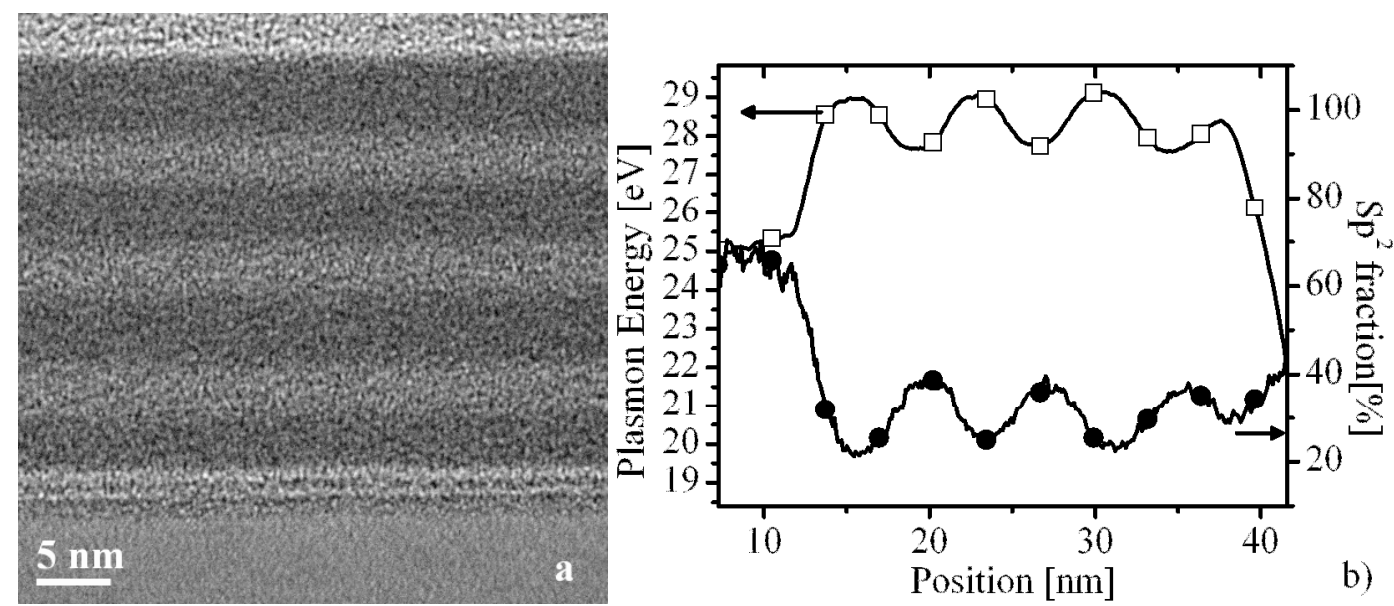

Figure 10a,b
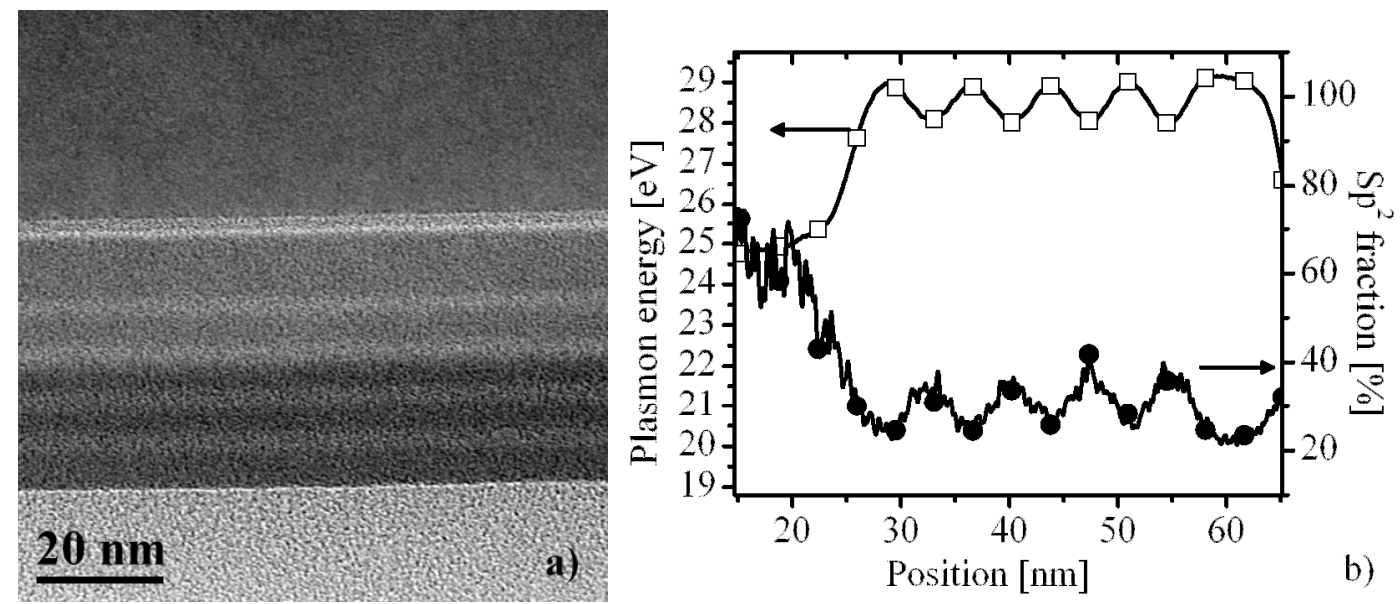
Figure 11

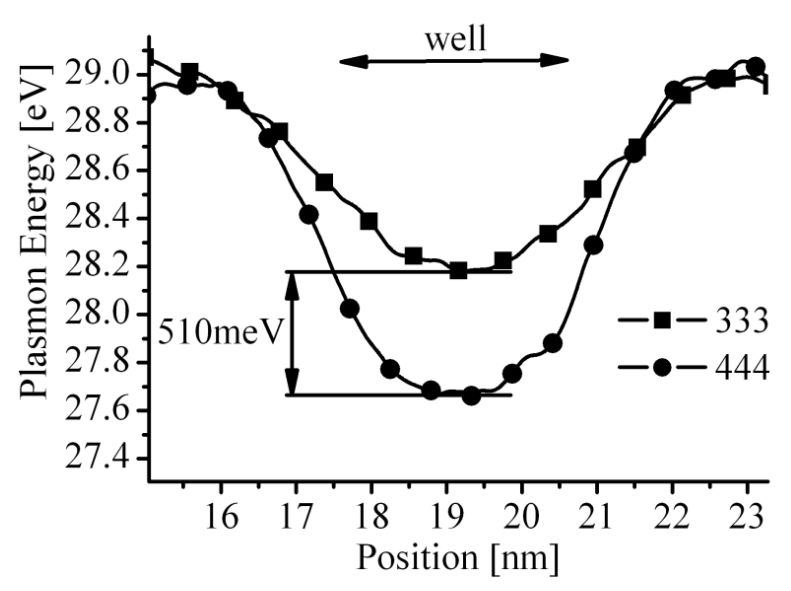


Figure captions:

Figure 1. a) Underfocused bright field image of an amorphous carbon superlattice, showing barriers as dark horizontal bands and wells as bright horizontal bands. The inset shows the area shown in the main image, and also identifies the position of the Si substrate. b) The equivalent ELSP image acquired at a $\sim 250 \mathrm{eV}$ offset, showing the distinctive sharp $1 \mathrm{~s}-\pi^{*}$ transition peak of the carbon K-edge. c) The equivalent ELSP image of the zero and low loss spectra of the superlattice in a). The Si and carbon plasmon peaks can be identified. The vertical dimensions in b) and c) are identical to that in a), to within a scaling factor $(\sim 0.5)$.

Figure 2. The spatial drift and fluctuation of the zero loss energy for the experimental set-up used. The values are measured from the drift corrections calculated over several ELSP image sets, acquired with $0.5 \mathrm{~s}$ exposures, $4.5 \mathrm{~s}$ apart.

Figure 3. Cut-out of the Si-superlattice interface in Figure 1c, showing the change in contrast at the interface, along the energy axis. This is caused by the chromatic aberration and the relative position of least contrast (indicated by arrow) can be changed with microscope defocus (Eq. 1). This allows us to determine $\mathrm{C}_{\mathrm{C}} \sim 1.5 \mathrm{~mm}$ for our experiment.

Figure 4. a) Detail of the plasmon energy traces across a superlattice at different relative microscope defoci, showing a loss of lattice 'contrast' with underfocus and overfocus, together with a change in the average plasmon energy, probably related to small changes in the collection angle with defocus. The inset shows the profiles across the whole superlattice, from the $\mathrm{Si}$ substrate (left) to the mounting adhesive (right). b) The lattice contrast, defined as the peak maxima and minima difference, normalised to their sum, shows an asymmetry between underfocus and overfocus. The interpolation is a guide to the eye.

Figure 5. The plasmon energy traces for different defoci (full lines) are compared with the 0nm defocus plasmon trace, convoluted by a 'loss of resolution' broadening Gaussian of varying FWHM (dashed lines with filled squares). The first number of the labels shows the defocus, whilst the second one shows the FWHM of the convoluted FWHM Gaussian.

Figure 6. The calculated plasmon energy in the middle of the sandwiched layer, as a function of the layer's thickness, for two collection angles. Negative thicknesses relate to a barrier-wellbarrier arrangement (bWb), whilst positive thicknesses describe the plasmon energy in the 
middle of the barrier of a well-barrier-well arrangement (wBw). For a 5mrad collection angle, the plasmon energy can be measured in layers of thicknesses down to $\sim 1 \mathrm{~nm}$ with some small correction. If the collection angle is increased to $\sim 50 \mathrm{mrad}$, then the plasmon energy can be measured, with some correction, for layer widths approaching $5 \AA$.

Figure 7. Comparison of the thickness and the plasmon energy profile across a superlattice. The observed preferential etching and the thickness gradient $\left(\sim 4^{\circ}\right)$ do not affect significantly the energy difference between barriers and wells. This is not the case when the thickness exceeds $0.5 \mathrm{t} / \lambda$, when removal of plural scattering is required.

Figure 8 a) Energy-filtered bright field image of the 888 ( $8 \mathrm{~nm}$ barrier- $8 \mathrm{~nm}$ well) superlattice (Si substrate at the bottom). b) the plasmon energy and $\mathrm{sp}^{2}$ fraction traces across the superlattice.

Figure 9 a) Energy-filtered bright field image of the 444 (4nm barrier-4nm well) superlattice ( $\mathrm{Si}$ substrate at the bottom). b) the plasmon energy and $\mathrm{sp}^{2}$ fraction traces across the superlattice.

Figure 10 a) Energy-filtered bright field image of the 333 ( $3 \mathrm{~nm}$ barrier-3nm well) superlattice (Si substrate at the bottom). b) the plasmon energy and $\mathrm{sp}^{2}$ fraction traces across the superlattice.

Figure 11. Comparison of the plasmon energy traces in the well of superlattices 444 (circles) and 333 (squares), showing a 510meV increase in the plasmon energy for the smaller well width. This could be interpreted as a $\sim 1 \mathrm{eV}$ increase in the Penn bandgap, due to quantum confinement of the barriers. 